Research Article

\title{
Theoretical Study of Synchronous Behavior in a Dual-Pendulum-Rotor System
}

\author{
Fang Pan $\left(\mathbb{D},{ }^{1}\right.$ Hou Yongjun $\mathbb{D}^{1},{ }^{1}$ Dai Liming $\mathbb{D},{ }^{2}$ and Du Mingjun $\mathbb{D}^{1}$ \\ ${ }^{1}$ School of Mechanical Engineering, Southwest Petroleum University, Chengdu, China \\ ${ }^{2}$ University of Regina, Regina, Canada \\ Correspondence should be addressed to Fang Pan; ckfangpan@126.com and Hou Yongjun; hyj2643446@126.com
}

Received 27 March 2018; Revised 16 May 2018; Accepted 4 June 2018; Published 9 July 2018

Academic Editor: Aly Mousaad Aly

Copyright (c) 2018 Fang Pan et al. This is an open access article distributed under the Creative Commons Attribution License, which permits unrestricted use, distribution, and reproduction in any medium, provided the original work is properly cited.

\begin{abstract}
A dual-pendulum-rotor system widely appears in aero-power plant, mining screening machines, parallel robots, and the like of the other rotation equipment. Unfortunately, the synchronous behavior related to the dual-pendulum-rotor system is less reported. Based on the special backgrounds, a simplified mechanical model of the dual-pendulum-rotor system is proposed in the paper, and the intrinsic mechanisms of synchronous phenomenon in the system are further revealed with employing the Poincaré method. The research results show that the spring stiffness, the installation angular of the motor, and rotation direction of the rotors have a large influence on the existence and stability of the synchronization state in the coupling system, and the mass ratios of the system are irrelevant to the synchronous state of the system. It should be noted that to ensure the implementation of the synchronization of the system, the values of the parameters of the system should be far away to the two "critical points".
\end{abstract}

\section{Introduction}

"Synchronization phenomenon" widely exists in nature and daily life. The so-called synchronization phenomenon refers to self-adjusting different frequencies of oscillating objects to a unified frequency relying on their internal weak couplings $[1,2]$. In recent years, the study of synchronization phenomenon is involved in the fields of physics, chemistry, and biology, such as the practical application on complex dynamic network systems [3-5], nonlinear coupled chaotic systems [6, 7], coupled pendulum system [8-12], and rotor system [1,1319]. Nevertheless, the latter two systems can be categorized as synchronous problem of mechanics system in detail. In the synchronous study of the pendulum system, antiphase selfsynchronization of clock pendulum is firstly discovered by Huygens in 1665. In the modern computation and analysis, the difference of spring stiffness influenced on the synchronous state is concerned, and the synchronization in inphase and anti-phase state for Huygens model is analyzed. Meanwhile, the derivatizations of Huygens model, including two coupled double pendula, coupling pendula under elastic forces, and pendula connected with linear springs, have attracted many scholars' attention. In the self-synchronous study of the rotor system, I.I.Blekhman proposed Poincaré method to explore the synchronous mechanism in multirotor systems. Nowadays, the Poincare method is still widely used in modern engineering design. Based on the foundation of I.I.Blekhman's method, many scientists proposed the other approaches to study self-synchronization of the rotor system. Wen Bangchun introduced an averaging method to calculate the synchronization and stability between multiple coupled rotors [13]. Zhao Chunyu [14, 15] gave a revised small parameter method to describe the synchronous process of rotor system, which greatly simplifies the solving process of synchronization problem in rotor system. Sperling presented a two-plane automatic balancing device for equilibration of rigid-rotor unbalance, on which the synchronization of the rigid rotors is determined with numerical method. Similarly, Balthazar [16] examined self-synchronization of four nonideal exciters in nonlinear vibration system via numerical simulations. Djanan. A. A. N [17] explored the system, three motors working on the same plate; the synchronous state depends on the physical characteristics of the motors and the plate. 


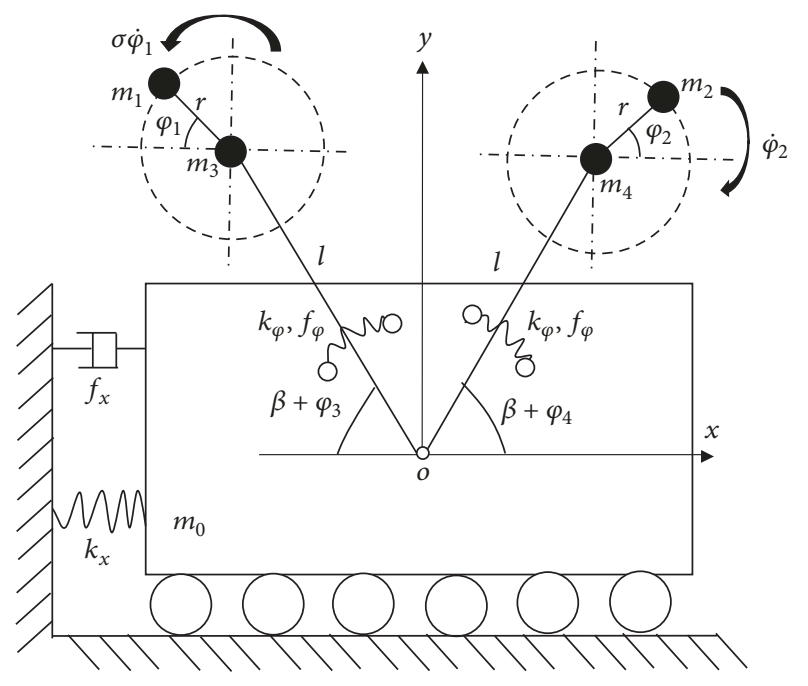

Figure 1: The dual-pendulum-rotor system.

The objects of the above-mentioned researches are mainly synchronization of the coupled pendula or the coupled rotors; however, the synchronization of the pendulumrotor system is less concerned. The extensive application of the pendulum-rotor system in aero-power plant, mining screening machines, parallel robots, and the like of the other rotation equipment is important for our industrial production and daily life [20-22]. The aim of this paper is to investigate a system composed of two unbalanced rotors coupled with two pendula in a horizontal plane. But mass of the motor and the rotor, which can be relatively heavy or light, may affect the system motion. Therefore, the proposed problem is different from the dynamics of classical pendulum systems presented in papers [8-11] and classical rotor systems presented in papers [12-20, 23-27]. We consider an ideal source of energy supply to asynchronous motors, which drag the unbalanced rotors. We also investigate selected aspects of the synchronization phenomenon. There are different synchronization states possible in mechanical systems. We will search for a complete synchronization when the rotors perform rotation with the difference direction or for the synchronous state when the phase differences between the rotors are stabilized.

\section{Dynamic Model}

A simplified dual-pendulum-rotor system is shown in Figure 1. This system consists of a rigid oscillating body of mass $m_{0}[\mathrm{Kg}]$ elastically supported via a linear damping spring with stiffness $k_{x}[\mathrm{~N} / \mathrm{m}]$ and damping $f_{x}[\mathrm{Ns} / \mathrm{m}]$. Unbalanced rotor $i$ actuated by an asynchronous motor is modelled by a point mass $m_{i}[\mathrm{Kg}]$ (for $i=1,2$ ) and attached at the end of a massless rod of length $r$ [m]; the rotation angle of the rotors is defined by $\varphi_{i} \in S^{1}$ (for $i=1,2$ ) in [rad]. It should be noted that all the motors are installed rigid vibrating body by linear torsion springs with stiffness $k_{\varphi}[\mathrm{N} / \mathrm{rad}]$ and damping $f_{\varphi}[\mathrm{N} \cdot \mathrm{m} /(\mathrm{rad} / \mathrm{s})]$. Thus, small oscillating should exist in the two motors when the motors are operated in steady state; the oscillating angle is denoted by $\varphi_{j} \in S^{1}$ (for $\left.j=3,4\right)$ in [rad]. Therefore, the oscillating characteristics of the motors are just like a pendulum or pendula with length $l$ and lumped mass (equal to the mass of the motors) $m_{j}[\mathrm{Kg}]$ (for $j=3,4$ ). The angular installation of the motors is denoted by $\beta \in S^{1}$ in [rad]; $x$ represents oscillation of centroid of the system in $\mathrm{x}$ direction, respectively. $\sigma$ is the rotation direction of the rotors; $l[\mathrm{~m}]$ is distance between pivot of the motor and centroid $O$ of the system.

In rotating coordinate $o^{\prime} x^{\prime \prime} y^{\prime \prime}$, the rotating centers of the rotors $\Phi_{1}^{\prime \prime}, \Phi_{2}^{\prime \prime}$ can be expressed as

$$
\begin{aligned}
& \boldsymbol{\Phi}_{1}^{\prime \prime}=\left(\begin{array}{c}
\sigma r \cos \varphi_{1}-l \cos \left(\varphi_{3}+\beta\right) \\
r \sin \varphi_{1}+l \sin \left(\varphi_{3}+\beta\right)
\end{array}\right), \\
& \boldsymbol{\Phi}_{2}^{\prime \prime}=\left(\begin{array}{c}
r \cos \varphi_{2}+l \cos \left(\varphi_{4}+\beta\right) \\
r \sin \varphi_{2}+l \sin \left(\varphi_{4}+\beta\right)
\end{array}\right) .
\end{aligned}
$$

And the rotating center of the motors $\Phi_{3}^{\prime \prime}$ and $\Phi_{4}^{\prime \prime}$ is represented as

$$
\begin{aligned}
& \boldsymbol{\Phi}_{3}^{\prime \prime}=\left(\begin{array}{c}
-l \cos \left(\beta+\varphi_{3}\right) \\
l \sin \left(\beta+\varphi_{3}\right)
\end{array}\right), \\
& \boldsymbol{\Phi}_{4}^{\prime \prime}=\left(\begin{array}{c}
l \cos \left(\beta+\varphi_{4}\right) \\
l \sin \left(\beta+\varphi_{4}\right)
\end{array}\right) .
\end{aligned}
$$

In fixed coordinate oxy, coordinates $\boldsymbol{\Phi}_{1}, \boldsymbol{\Phi}_{2}, \boldsymbol{\Phi}_{3}, \boldsymbol{\Phi}_{4}$ can be obtained by

$$
\Phi_{i}=\Phi_{0}+\Phi_{i}^{\prime \prime} . \quad(i=1,2,3,4)
$$

where $\boldsymbol{\Phi}_{0}$ is displacement vector of the vibrating base, $\boldsymbol{\Phi}_{0}=$ $[x, 0]^{\mathrm{T}}$.

According to kinetic theory, kinetic energy $\mathrm{T}$ of the dualpendulum-rotor system should be written as follows:

$$
T=\frac{1}{2} m_{0} \dot{x}+\frac{1}{2} \sum_{i=1}^{4} m_{i} \dot{\Phi}_{i}^{\mathrm{T}} \dot{\Phi}_{i}+\frac{1}{2} \sum_{i=1}^{2} J_{i} \dot{\varphi}_{i}^{2}
$$

$J_{1}$ and $J_{2}$ are the rotational inertia of the rotors, and symbols $(\bullet)$ and $(\bullet)$ denote $d(\bullet) / d t$ and $d^{2}(\bullet) / d t^{2}$, respectively.

In addition, potential energy $V$ of the dual-pendulumrotor system can be expressed by

$$
V=\frac{1}{2} k_{x} x^{2}+\frac{1}{2} k_{\varphi} \varphi_{3}^{2}+\frac{1}{2} k_{\varphi} \varphi_{4}^{2}
$$

Moreover, dissipated energy $D$ of the system can be obtained by

$$
D=\frac{1}{2} f_{x} \dot{x}^{2}+\frac{1}{2} f_{1} \dot{\varphi}_{1}^{2}+\frac{1}{2} f_{2} \dot{\varphi}_{2}^{2}+\frac{1}{2} f_{3} \dot{\varphi}_{3}^{2}+\frac{1}{2} f_{4} \dot{\varphi}_{4}^{2}
$$

According to the Lagrange equation

$$
\frac{d}{d t}\left(\frac{\partial T}{\partial \dot{q}}\right)-\frac{\partial(T-V)}{\partial q}+\frac{\partial D}{\partial q}=Q_{i}
$$


the dynamic equation of the dual-pendulum-rotor system can be derived. In the system, coordinate matrix $\mathbf{q}=$ $\left[x, \varphi_{1}, \varphi_{2}, \varphi_{3}, \varphi_{4}\right]^{\mathrm{T}}$ is considered as the generalized coordinate matrix; meanwhile, the generalized force matrix of the system is assumed as

$$
\begin{aligned}
& {\left[\begin{array}{lllll}
Q_{\mathrm{x}} & Q_{\varphi_{1}} & Q_{\varphi_{2}} & Q_{\varphi_{3}} & Q_{\varphi_{4}}
\end{array}\right]^{\mathrm{T}}} \\
& \quad=\left[\begin{array}{lllll}
0 & M_{e 1}-R_{e 1} & M_{e 2}-R_{e 2} & 0 & 0
\end{array}\right]^{\mathrm{T}}
\end{aligned}
$$

Substituting (4), (5), (6), and (8) into (7) and, meanwhile, considering $m_{1}, m_{2} \ll m_{0}, \sum_{k=0}^{4} m_{k}=M$. As $J_{1}$ and $J_{2}$ are sums of rotational inertia of motor's rotor about spin axis and that of eccentric lump, compared with $m_{1} r^{2}$ and $m_{1} r^{2}$, the rotational inertia of motor's rotor about spin axis is too small to be neglected; i.e., $J_{1} \approx m_{1} r^{2}, J_{2} \approx m_{2} r^{2}$, and the dynamic equation of the dual-pendulum-rotor system is written by

$$
\begin{gathered}
J_{1} \ddot{\varphi}_{1}=M_{e 1}-R_{e 1}+\sigma m_{1} r \sin \varphi_{1} \ddot{x} \\
-m_{1} r l \ddot{\varphi}_{3} \cos \left(\beta+\sigma \varphi_{1}\right) \\
+m_{1} r l \dot{\varphi}_{3}^{2} \sin \left(\beta+\sigma \varphi_{1}\right) \\
J_{2} \ddot{\varphi}_{2}+J_{2} \ddot{\varphi}_{2}=M_{e 2}-R_{e 2}+m_{2} r \sin \varphi_{2} \ddot{x} \\
-m_{2} r l \ddot{\varphi}_{4} \cos \left(\beta-\varphi_{2}\right) \\
+m_{2} r l \dot{\varphi}_{4}^{2} \sin \left(\beta-\varphi_{2}\right) \\
M \ddot{x}+f_{x} \dot{x}+k_{x} x \\
=\sigma m_{1} r\left(\ddot{\varphi}_{1} \sin \varphi_{1}+\dot{\varphi}_{1}^{2} \cos \varphi_{1}\right) \\
+m_{2} r\left(\ddot{\varphi}_{2} \sin \varphi_{2}+\dot{\varphi}_{2}^{2} \cos \varphi_{2}\right) \\
-\left(m_{1}+m_{3}\right) l\left(\ddot{\varphi}_{3} \sin \beta+\dot{\varphi}_{3}^{2} \cos \beta\right) \\
+\left(m_{2}+m_{4}\right) l\left(\ddot{\varphi}_{4} \sin \beta+\dot{\varphi}_{4}^{2} \cos \beta\right) \\
\left(m_{2} l^{2}+m_{4} l^{2}\right) \ddot{\varphi}_{4}+f_{\varphi} \dot{\varphi}_{4}+k_{\varphi} \varphi_{4} \\
=\left(m_{2}+m_{4}\right) l \sin \beta \ddot{x} \\
-m_{2} l r\left[\ddot{\varphi}_{2} \cos \left(\beta-\varphi_{2}\right)+\dot{\varphi}_{2}^{2} \sin \left(\beta-\varphi_{2}\right)\right] \\
\left.=m_{1} l^{2}\right) \ddot{\varphi}_{3}+f_{\varphi} \dot{\varphi}_{3}+k_{\varphi} \varphi_{3} \\
\left.+m_{3}\right) l \sin \beta \ddot{x}
\end{gathered}
$$

where

$\sigma$ $= \begin{cases}1, & \text { the two rotor rotate in the same direction, } \\ -1, & \text { the two rotor rotate in the opposite direction. }\end{cases}$
It can be seen that the terms of $\dot{\varphi}_{3}^{2}$ and $\dot{\varphi}_{4}^{2}$, nonlinear terms owing to the small periodic vibration in synchronous state, are included in (9c). The terms related to $\dot{\varphi}_{3}^{2}$ and $\dot{\varphi}_{4}^{2}$ can be rewritten as Poincaré style with small parameter $\mu$. Meanwhile, damping coefficient of this system is very small, and so the terms with damping coefficient can be rearranged as Poincaré style. In synchronous state, velocity of the motors is fluctuated slightly and periodically; hence, the acceleration of the rotors is also considered as a small term. Therefore, introducing small parameter $\mu$, the Poincaré style of (9a), $(9 b),(9 c),(9 d),(9 e)$ is given as follows [27]:

$$
\begin{gathered}
J_{1} \ddot{\varphi}_{1}=\mu \Phi_{1} \\
J_{2} \ddot{\varphi}_{2}=\mu \Phi_{2} \\
M \ddot{x}+k_{x} x=\sigma m_{1} r\left(\ddot{\varphi}_{1} \sin \varphi_{1}+\dot{\varphi}_{1}^{2} \cos \varphi_{1}\right) \\
+m_{2} r\left(\ddot{\varphi}_{2} \sin \varphi_{2}+\dot{\varphi}_{2}^{2} \cos \varphi_{2}\right)-\left(m_{1}+m_{3}\right) l \ddot{\varphi}_{3} \\
\cdot \sin \beta+\left(m_{2}+m_{4}\right) l \ddot{\varphi}_{4} \sin \beta-\mu\left[f_{x} \dot{x}\right. \\
\left.+\left(m_{1}+m_{3}\right) l \dot{\varphi}_{3}^{2} \cos \beta-\left(m_{2}+m_{4}\right) l \dot{\varphi}_{4}^{2} \cos \beta\right] \\
\left(m_{1} l^{2}+m_{3} l^{2}\right) \ddot{\varphi}_{3}+k_{\varphi} \varphi_{3} \\
=-\left(m_{1}+m_{3}\right) l \sin \beta \ddot{x}-m_{1} l r \ddot{\varphi}_{1} \cos \left(\beta+\sigma \varphi_{1}\right) \\
-\mu\left[f_{\varphi} \dot{\varphi}_{3}-\sigma m_{1} l r \dot{\varphi}_{1}^{2} \sin \left(\beta+\sigma \varphi_{1}\right)\right] \\
\left(m_{2} l^{2}+m_{4} l^{2}\right) \ddot{\varphi}_{4}+k_{\varphi} \varphi_{4} \\
=\left(m_{2}+m_{4}\right) l \sin \beta \ddot{x}-m_{2} l r \ddot{\varphi}_{2} \cos \left(\varphi_{2}-\beta\right) \\
-\mu\left[f_{\varphi} \dot{\varphi}_{4}+m_{2} l r \dot{\varphi}_{2}^{2} \sin \left(\varphi_{2}-\beta\right)\right]
\end{gathered}
$$

where

$$
\begin{aligned}
\mu \Phi_{1}= & M_{e 1}-R_{e 1}+\sigma m_{1} r \sin \varphi_{1} \ddot{x} \\
& -m_{1} r l \ddot{\varphi}_{3} \cos \left(\beta+\sigma \varphi_{1}\right) \\
& +m_{1} r l \dot{\varphi}_{3}^{2} \sin \left(\beta+\sigma \varphi_{1}\right) \\
\mu \Phi_{2}= & M_{e 2}-R_{e 2}+m_{2} r \sin \varphi_{2} \ddot{x} \\
& -m_{2} r l \ddot{\varphi}_{4} \cos \left(\beta-\varphi_{2}\right) \\
& +m_{2} r l \dot{\varphi}_{4}^{2} \sin \left(\beta-\varphi_{2}\right)
\end{aligned}
$$

Based on Zhao's method [15], the electromagnetic torque of the asynchronous motor is linearized at stable point $\dot{\varphi}_{i}=\omega_{s}$ when the two motors are synchronously operated; i.e.,

$$
M_{e i}=n_{p} \frac{L_{m i}^{2} U_{S 0}^{2}}{L_{s i}^{2} \omega_{m} R_{r i}}\left(\omega_{s}-n_{p} \omega_{m}\right) \quad(i=1,2)
$$

where $L_{m i}$ is coefficient of mutual asynchronous in the $i$ th motor; $L_{s i}$ is the $i$ th coefficient of stator inductance in the $i$ th motor; $n_{p}$ is pole-pairs of the motors; $\omega_{m}$ is synchronous velocity of the motors; $R_{r i}$ is rotor resistance in the $i$ th motor; $U_{S 0}$ is amplitude of stator voltage. 


\section{Approximate Solution}

From formulas (9a) to (9e), it can be seen that the vibration characteristics of the system are coupled related to DOFs $\varphi_{1}, \varphi_{2}, x, \varphi_{3}$, and $\varphi_{4}$. Neglecting the terms of small parameters, introduce the dimensionless parameter as follows:

$$
\begin{aligned}
\frac{r}{l} & =r_{l}, \\
\frac{m}{M} & =r_{m}, \\
\frac{m_{i}}{m} & =\eta_{i} \quad(i=1,2,3, \ldots, 4), \\
\omega_{x} & =\sqrt{\frac{k_{x}}{M}}, \\
\omega_{\varphi} & =\sqrt{\frac{k_{\varphi}}{m_{1} l^{2}}+m_{3} l^{2},} \\
\omega_{\varphi} & =\sqrt{k_{\varphi} / m_{1} l^{2}+m_{3} l^{2}}, \\
f_{1}(t) & =r_{m} r_{l}\left(\sigma \eta_{1} \cos \varphi_{1}+\eta_{2} \cos \varphi_{2}\right), \\
f_{2}(t) & =\frac{\eta_{1} r_{l}}{\left(\eta_{1}+\eta_{3}\right)} \sin \left(\varphi_{1}+\sigma \beta\right), \\
f_{3}(t) & =\frac{\eta_{2} r_{l}}{\left(\eta_{2}+\eta_{4}\right)} \sin \left(\varphi_{2}-\beta\right) .
\end{aligned}
$$

In addition, velocities $\dot{\varphi}_{1}$ and $\dot{\varphi}_{2}$ of the two motors approached to a constant are represented by $\omega_{m}$ in the steady state; therefore, the accelerations of the two motors should be equal to zero; i.e., $\ddot{\varphi}_{1}=0$ and $\ddot{\varphi}_{2}=0$. In this case, (11c) -(11e) can be simplified as the dimensionless style:

$$
\begin{gathered}
\ddot{x}+\omega_{x}^{2} x=\omega_{m}^{2} l f_{1}(t)-\left(\eta_{1}+\eta_{3}\right) r_{m} l \sin \beta \ddot{\varphi}_{3} \\
+\left(\eta_{2}+\eta_{4}\right) r_{m} l \sin \beta \ddot{\varphi}_{4}, \\
\ddot{\varphi}_{3}+\omega_{\varphi}^{2} \varphi_{3}=-\frac{\sin \beta}{l} \ddot{x}+\omega_{m}^{2} f_{2}(t), \\
\ddot{\varphi}_{4}+{\underset{\sim}{\omega}}_{\varphi}^{2} \varphi_{4}=\frac{\sin \beta}{l} \ddot{x}+\omega_{m}^{2} f_{3}(t) .
\end{gathered}
$$

For simplifying the solution procedure, consider that the natural frequencies of the torsion spring are quasi-identical; thus, parameter ${\underset{\sim}{\omega}}_{\varphi}$ should be replaced by $\omega_{\varphi}$.

Applying Laplace transformation in (16a), (16b), (16c), the coupling terms can be decoupled. Therefore, we have

$$
\begin{aligned}
& s^{2} X+\omega_{x}^{2} X= \omega_{m}^{2} l F_{1}(s)-\left(\eta_{1}+\eta_{3}\right) r_{m} l s^{2} \sin \beta \phi_{3} \\
&+\left(\eta_{2}+\eta_{4}\right) r_{m} l s^{2} \sin \beta \phi_{4} \\
& s^{2} \phi_{3}+ \omega_{\varphi}^{2} \phi_{3}=-\frac{s^{2} \sin \beta}{l} X+\omega_{m}^{2} F_{2}(s) \\
& s^{2} \phi_{4}+\omega_{\varphi}^{2} \phi_{4}=\frac{s^{2} \sin \beta}{l} X+\omega_{m}^{2} F_{3}(s)
\end{aligned}
$$

From formulas (16a)-(16c), the approximate solutions of the system in complex domain can be given by

$$
\begin{aligned}
X= & \frac{\omega_{m}^{2} l}{G_{1}(s)} F_{1}(s)-\frac{\left(\eta_{1}+\eta_{3}\right) \omega_{m}^{2} r_{m} l s^{2} \sin \beta}{G_{1}(s)} F_{2}(s) \\
& +\frac{\left(\eta_{2}+\eta_{4}\right) \omega_{m}^{2} r_{m} l s^{2} \sin \beta}{G_{1}(s)} F_{3}(s) \\
\phi_{3}= & -\frac{s^{2} \sin \beta \omega_{m}^{2}}{G_{1}(s)} F_{1}(s) \\
& +\left[\frac{\omega_{m}^{2}}{G_{2}(s)}+\frac{\omega_{m}^{2}\left(\eta_{1}+\eta_{3}\right) r_{m} s^{4} \sin ^{2} \beta}{G_{1}(s) G_{2}(s)}\right] F_{2}(s) \\
& -\frac{\omega_{m}^{2}\left(\eta_{2}+\eta_{4}\right) r_{m} s^{4} \sin ^{2} \beta}{G_{1}(s) G_{2}(s)} F_{3}(s) \\
\phi_{4}= & \frac{\omega_{m}^{2} s^{2} \sin \beta}{G_{1}(s)} F_{1}(s) \\
& +\frac{\omega_{m}^{2}\left(\eta_{1}+\eta_{3}\right) r_{m} s^{4} \sin ^{2} \beta}{G_{1}(s) G_{2}(s)} F_{2}(s) \\
& {\left[\frac{\omega_{m}^{2}}{G_{2}(s)}+\frac{\omega_{m}^{2}\left(\eta_{2}+\eta_{4}\right) r_{m} s^{4} \sin ^{2} \beta}{G_{1}(s) G_{2}(s)}\right] F_{3}(s) }
\end{aligned}
$$

where $G_{1}(s)=\left(s^{2}+\omega_{x}^{2}\right)\left(s^{2}+\omega_{\varphi}^{2}\right)-\left(\eta_{1}+\eta_{3}+\eta_{2}+\right.$ $\left.\eta_{4}\right) r_{m} s^{4} \sin ^{2} \beta, G_{2}(s)=s^{2}+\omega_{\varphi}^{2}$.

Then, the numerators and denominators of (17a), (17b), (17c) in the right are divided by divisor $\omega_{\varphi}{ }^{4} \omega_{x}{ }^{2}$; moreover, introduce frequency ratios

$$
\begin{aligned}
& n_{x}=\frac{\omega_{m}}{\omega_{x}}, \\
& n_{\varphi}=\frac{\omega_{m}}{\omega_{\varphi}}
\end{aligned}
$$

into (17a)-(17c). In this case, the coefficients of spring stiffness $k_{x}$ and $k_{\varphi}$ are converted into the frequency ratios $n_{x}$ and $n_{\varphi}$ in light of (14), respectively.

Finally, through applying the inverse Laplace transformation to (18), the approximate solutions of the system in time domain can be obtained by

$$
\begin{aligned}
x= & \mu_{11} l f_{1}(t)+\mu_{12}\left(\eta_{1}+\eta_{3}\right) l r_{m} f_{2}(t) \\
& +\mu_{13}\left(\eta_{2}+\eta_{4}\right) l r_{m} f_{3}(t), \\
\varphi_{3}= & \mu_{21} f_{1}(t)+\mu_{22}\left(\eta_{1}+\eta_{3}\right) r_{m} f_{2}(t) \\
& +\mu_{23}\left(\eta_{2}+\eta_{4}\right) r_{m} f_{3}(t), \\
\varphi_{4}= & \mu_{31} f_{1}(t)+\mu_{32}\left(\eta_{1}+\eta_{3}\right) r_{m} f_{2}(t) \\
& +\mu_{33}\left(\eta_{2}+\eta_{4}\right) r_{m} f_{3}(t),
\end{aligned}
$$


where

$$
\begin{aligned}
\mu_{11}= & \frac{n_{x}^{2}\left(1-n_{\varphi}^{2}\right)}{g}, \\
\mu_{12}= & \mu_{21}=\frac{n_{x}^{2} n_{\varphi}^{2} \sin \beta}{g}, \\
\mu_{13}= & \mu_{31}=-\frac{n_{x}^{2} n_{\varphi}^{2} \sin \beta}{g}, \\
\mu_{22}= & \mu_{33}=\frac{n_{x}^{2} n_{\varphi}^{4} \sin ^{2} \beta}{\left(1-n_{\varphi}^{2}\right) g}+\frac{n_{\varphi}^{2}}{\left(1-n_{\varphi}^{2}\right)\left(\eta_{1}+\eta_{3}\right) r_{m}}, \\
\mu_{23}= & \mu_{32}=-\frac{n_{x}^{2} n_{\varphi}^{4} \sin ^{2} \beta}{\left(1-n_{\varphi}^{2}\right) g}, \\
g= & \left(1-n_{x}^{2}\right)\left(1-n_{\varphi}^{2}\right) \\
& -\left(\eta_{1}+\eta_{3}+\eta_{2}+\eta_{4}\right) r_{m} n_{x}^{2} n_{\varphi}^{2} \sin ^{2} \beta
\end{aligned}
$$

In light of functions $f_{1}(t), f_{2}(t)$, and $f_{3}(t)$ in (14), the approximate solutions of the system can be rearranged as the following:

$$
\begin{aligned}
x= & \sigma \mu_{11} \eta_{1} r r_{m} \cos \varphi_{1}+\mu_{11} \eta_{2} r r_{m} \cos \varphi_{2} \\
& +\mu_{12} \eta_{1} r r_{m} \sin \left(\varphi_{1}+\sigma \beta\right) \\
& +\mu_{13} \eta_{2} r r_{m} \sin \left(\varphi_{2}-\beta\right), \\
\varphi_{3}= & \sigma \mu_{21} \eta_{1} r_{m} r_{l} \cos \varphi_{1}+\mu_{21} \eta_{2} r_{m} r_{l} \cos \varphi_{2} \\
& +\mu_{22} \eta_{1} r_{l} r_{m} \sin \left(\varphi_{1}+\sigma \beta\right) \\
& +\mu_{23} \eta_{2} r_{l} r_{m} \sin \left(\varphi_{2}-\beta\right), \\
\varphi_{4}= & \mu_{31} r_{m} r_{l}\left(\sigma \eta_{1} \cos \varphi_{1}+\eta_{2} \cos \varphi_{2}\right) \\
& +\mu_{32} \eta_{1} r_{l} r_{m} \sin \left(\varphi_{1}+\sigma \beta\right) \\
& +\mu_{33} \eta_{2} r_{l} r_{m} \sin \left(\varphi_{2}-\beta\right) .
\end{aligned}
$$

\section{Synchronization}

Based on the approximate solutions above, the synchronization and stability of the system can be determined with Poincaré method [20, 25-27]. In addition, the phase angle of the rotors can be assumed by

$$
\begin{aligned}
& \varphi_{1}=\omega_{m} t+\alpha_{1} \\
& \varphi_{2}=\omega_{m} t+\alpha_{2}
\end{aligned}
$$

Substituting (22) into (21a), (21b), (21c), the approximate solutions of the system can be written by

$$
\begin{aligned}
x= & \mu_{11} r r_{m}\left[\sigma \eta_{1} \cos \left(\omega_{m} t+\alpha_{1}\right)+\eta_{2} \cos \left(\omega_{m} t+\alpha_{2}\right)\right] \\
& +\mu_{12} r r_{m} \eta_{1} \sin \left(\omega_{m} t+\alpha_{1}+\sigma \beta\right) \\
& +\mu_{13} r r_{m} \eta_{2} \sin \left(\omega_{m} t+\alpha_{2}-\beta\right),
\end{aligned}
$$

$\varphi_{3}$

$$
\begin{aligned}
= & \mu_{21} r_{m} r_{l}\left[\sigma \eta_{1} \cos \left(\omega_{m} t+\alpha_{1}\right)+\eta_{2} \cos \left(\omega_{m} t+\alpha_{2}\right)\right] \\
& +\mu_{22} r_{l} r_{m} \eta_{1} \sin \left(\omega_{m} t+\alpha_{1}+\sigma \beta\right) \\
& +\mu_{23} r_{l} r_{m} \eta_{2} \sin \left(\omega_{m} t+\alpha_{2}-\beta\right), \\
\varphi_{4} & \\
= & \mu_{31} r_{m} r_{l}\left[\sigma \eta_{1} \cos \left(\omega_{m} t+\alpha_{1}\right)+\eta_{2} \cos \left(\omega_{m} t+\alpha_{2}\right)\right] \\
& +\mu_{32} \eta_{1} r_{l} r_{m} \sin \left(\omega_{m} t+\alpha_{1}+\sigma \beta\right) \\
& +\mu_{33} \eta_{2} r_{l} r_{m} \sin \left(\omega_{m} t+\alpha_{2}-\beta\right) .
\end{aligned}
$$

$\varphi_{4}$

As the values of the phase difference of the rotors may be different when the asynchronous motors are operated in the steady state, the phase difference between the rotors is noted by

$$
\alpha=\alpha_{1}-\alpha_{2}
$$

The second derivatives of $x, \varphi_{3}$, and $\varphi_{4}$ with respect to time $t$ can be obtained. Substituting $\ddot{x}, \ddot{\varphi}_{3}$, and $\ddot{\varphi}_{4}$ into (12a) and (12b), and then integrating and averaging these equations related to $t$ over period $T$, respectively, we can obtain

$$
\begin{aligned}
& P_{1}=\frac{1}{T} \int_{0}^{T} \mu \Phi_{1} d t=\bar{M}_{e 1}-\bar{R}_{e 1}+\frac{1}{2} \\
& \cdot \eta_{1} \eta_{2} m r^{2} \omega_{m}^{2} r_{m}\left\{\begin{array}{c}
-\sigma \mu_{11} \sin \alpha+\sigma \mu_{12} \cos (\alpha+\beta) \\
+\mu_{21} \cos (\alpha+\sigma \beta)-\mu_{23} \sin [\alpha+(\sigma+1) \beta]
\end{array}\right\} \\
& P_{2}=\frac{1}{T} \int_{0}^{T} \mu \Phi_{2} d t=\bar{M}_{e 2}-\bar{R}_{e 2}+\frac{1}{2} \\
& \cdot \eta_{1} \eta_{2} m r^{2} \omega_{m}^{2} r_{m}\left\{\begin{array}{c}
-\sigma \mu_{11} \sin \alpha+\sigma \mu_{12} \cos (\alpha+\beta) \\
+\mu_{21} \cos (\alpha+\sigma \beta)-\mu_{23} \sin [\alpha+(\sigma+1) \beta]
\end{array}\right\}
\end{aligned}
$$

$P_{1}$ and $P_{2}$ represent the averaging residual torque of the first and second motor in single period $T$, respectively. As we all know, when the motors are operated in synchronous state, $\mu$ is a small parameter approached to zero. Therefore, the residual torque in the motors is approximated to zero; i.e.,

$$
P_{1} \approx P_{2} \approx 0
$$

Substituting (27) into (25) and (26), the balanced torques of the motor are written by

$$
\begin{aligned}
& \bar{M}_{e 1}-\bar{R}_{e 1}=\frac{1}{2} \eta_{1} \eta_{2} m r^{2} \omega_{m}^{2} r_{m}\left\{\sigma \mu_{11} \sin \alpha\right. \\
& -\sigma \mu_{12} \cos (\alpha+\beta)-\mu_{21} \cos (\alpha+\sigma \beta) \\
& \left.+\mu_{23} \sin [\alpha+(\sigma+1) \beta]\right\} \\
& \bar{M}_{e 2}-\bar{R}_{e 2}=\frac{1}{2} \eta_{1} \eta_{2} m r^{2} \omega_{m}^{2} r_{m}\left\{-\sigma \mu_{11} \sin \alpha\right. \\
& +\sigma \mu_{12} \cos (\alpha+\beta)+\mu_{21} \cos (\alpha+\sigma \beta) \\
& \left.-\mu_{23} \sin [\alpha+(\sigma+1) \beta]\right\}
\end{aligned}
$$


$\bar{M}_{e i}, \bar{R}_{e i}(i=1,2)$ represent average electromagnetic and friction torque in the motors over period $T$, respectively. When the two rotors are operated synchronously, the vibrating system transmits electromagnetic torque between the two motors to overcome the differences of output torque for the two rotors by adjusting the phase difference between two rotors. The differences of output torque $\Delta M$ between the motors can be expressed as

$$
\Delta M=\bar{M}_{e 1}-\bar{R}_{e 1}-\left(\bar{M}_{e 2}-\bar{R}_{e 2}\right)=\eta_{1} \eta_{2} m r^{2} \omega_{m}^{2} r_{m}\left\{\begin{array}{c}
\sigma \mu_{11} \sin \alpha-\sigma \mu_{12} \cos (\alpha+\beta)-\mu_{21} \cos (\alpha+\sigma \beta) \\
\sigma \mu_{12} \cos (\alpha+\beta)+\mu_{21} \cos (\alpha+\sigma \beta)
\end{array}\right\}
$$

As the parameter of the two asynchronous motors is identical, the differences of output torque should be zero. Therefore, to implement synchronous operation, the values of the parameters in the system must satisfy the following equation:

$$
\begin{gathered}
-\sigma \mu_{11} \sin \alpha+\sigma \mu_{12} \cos (\alpha+\beta)+\mu_{21} \cos (\alpha+\sigma \beta) \\
-\mu_{23} \sin [\alpha+(\sigma+1) \beta]=0
\end{gathered}
$$

which is called balance equation of synchronous state. Obviously, the phase difference is influenced by the rotation direction of the motors, the coupling coefficients between rotor and pendula, and the installation location of the motors. According to (30), we can determine phase difference $\alpha$ with numerical computations. With trigonometric function, the equation above can be expanded as

$$
\begin{aligned}
& \cot \alpha \\
& =\frac{\sigma \mu_{11}+\sigma \mu_{12} \sin \beta+\sigma \mu_{21} \sin \beta+\mu_{23} \cos [(\sigma+1) \beta]}{\sigma \mu_{12} \cos \beta+\mu_{21} \cos \beta-\mu_{23} \sin [(\sigma+1) \beta]}
\end{aligned}
$$

If some solutions of phase difference $\alpha$ exist in (31), the denominator of this equality in the right should be nonzero. Define the denominator of this equality with a symbol $D$; i.e.,

$$
D=\sigma \mu_{12} \cos \beta+\mu_{21} \cos \beta-\mu_{23} \sin [(\sigma+1) \beta]
$$

It can be known that denominator $D$ is a function related to variables $\sigma, \mu_{12}, \mu_{21}, \mu_{23}$, and $\beta$, but parameter $\sigma$ indicates the rotation directions of the motors independent of nonzero solution of denominator $D$. Therefore, we will discuss zero solution of parameter $D$ related to installation angle and coupling coefficients. There are two cases causing the zero solution of denominator $D$.

(1) Parameter $\beta$ represents installation angle of the motors. Thus, parameter $\beta$ with a particular value leads to $D$ being zero. The first "critical point" is

$$
\beta=k \pi-\pi, \quad(k=1,2,3 \ldots) .
$$

When the installation angles of the motors are approximated or equal to this point, the absent-synchronization of the system will be implemented. In this situation, the vibrating characteristics of the oscillating body are unascertainable.

(2) According to (20), coupling coefficients $\mu_{12}, \mu_{21}$, and $\mu_{23}$ are fractions; thus parameters $n_{x}$ and $n_{\varphi}$ with particular values lead to $D$ being zero. The second "critical point" is

$$
n_{x}=1 \text { or } n_{\varphi}=1
$$

The second "critical point" means that the excitation frequency of the motors is identical to the natural frequency of the springs. In this case, strong resonance exists in such value of the frequency ratios. However, frequency ratios $n_{x}$ and $n_{\varphi}$ are the function related to stiffness coefficients $k_{x}$ and $k_{\varphi}$ of the springs, which indicates that stiffness coefficients are the key parameters to determine phase difference $\alpha$.

Clearly, to ensure the synchronization and stability of the system, the value of frequency ratios $n_{x}$ and $n_{\varphi}$ should be far away from 1 . In light of different value of $n_{\varphi}$ and $n_{\varphi}$, the coupling type of the system can be defined as follows:

Type 1: system of before-resonance coupled beforeresonance $\left(0.1<n_{x}<1\right.$ and $\left.0.1<n_{\varphi}<1\right)$.

Type 2: system of after-resonance coupled beforeresonance $\left(0.1<n_{x}<1\right.$ and $1.0<n_{\varphi}<7$, or $1.0<n_{x}<7$ and $\left.0.1<n_{\varphi}<1\right)$.

Type 3: system of after-resonance coupled after-resonance $\left(1.0<n_{\varphi}<7\right.$ and $\left.1.0<n_{x}<7\right)$.

Eliminating the two kinds of "critical point", the phase difference between the two rotors can be calculated:

$\alpha$

$$
=\operatorname{arccot} \frac{\sigma \mu_{11}+\sigma \mu_{12} \sin \beta+\sigma \mu_{21} \sin \beta+\mu_{23} \cos [(\sigma+1) \beta]}{\sigma \mu_{12} \cos \beta+\mu_{21} \cos \beta-\mu_{23} \sin [(\sigma+1) \beta]} .
$$

Obviously, the value of the phase difference is related to the coupling coefficients $\left(\mu_{11}, \mu_{12}, \mu_{21}\right.$, and $\left.\mu_{23}\right)$ and the installation angle. Coupling coefficients represent the mutual coupling ability among the oscillating body, rotors, and pendula through the springs. The larger the coupling coefficients are, the stronger the coupling ability of the system is. Obviously, coupling coefficients $\mu_{12}, \mu_{21}$, and $\mu_{23}$ contain $\sin \beta$; thus the absence of the coupling ability appears when $\beta=0$. In this situation, the rotors cannot implement synchronous operation. It should be noted that coupling coefficients are the functions of parameters $n_{x}, n_{\varphi}, \beta$, and $r_{m}$, and these parameters may influence the value of phase difference $\alpha$.

\section{Synchronous Stability}

From (35), some values of phase difference can be obtained; however, the stability of these values should be determined as I.I.Blekhman's method $[1,2]$. If a certain constant $\alpha=\alpha^{*}$ satisfies equation

$$
\frac{\partial\left(P_{1}-P_{2}\right)}{\partial \alpha}-\chi=0
$$


TABLE 1: Parameter values.

(a) Parameter values for system equation (6)

\begin{tabular}{lccc}
\hline Unbalanced rotor for $i=1,2$ & Vibroplatform & Pendulum rod & Asynchronous motor \\
\hline$m_{i}=2[\mathrm{~kg}]$ & $m_{0}=100[\mathrm{~kg}]$ & $l=0.3[\mathrm{~m}]$ & $m_{3}=m_{4}=14[\mathrm{~kg}]$ \\
$r=0.05[\mathrm{~m}]$ & $k_{x}=246490000-50307[\mathrm{~N} / \mathrm{m}]$ & $k_{\varphi}=4980-24402510[\mathrm{Nm} / \mathrm{rad}]$ & $L_{m i}=0.13[\mathrm{H}]$ \\
$\omega_{m}=152-157[\mathrm{rad} / \mathrm{s}]$ & $f_{x}=1064[\mathrm{Ns} / \mathrm{m}]$ & $f_{\varphi}=15[\mathrm{Nm} /(\mathrm{rad} / \mathrm{s})]$ & $L_{s i}=0.1[\mathrm{H}]$ \\
- & - & $\beta=0-5 \pi / 12[\mathrm{rad}]$ & $n_{p}=2$ \\
- & - & - & $R_{r i}=0.54[\Omega]$ \\
- & - & - & $U_{S 0}=220[\mathrm{~V}]$ \\
\hline
\end{tabular}

(b) Parameter values according to dimensionless equations (11a), (11b), (11c), (11d), (1le)

$$
\begin{gathered}
\eta_{1}=0.2,1 \\
\eta_{2}=1 \\
\eta_{3}=\eta_{4}=12 \\
\sigma=-1,1 \\
r_{m}=0.02 \\
n_{x}=0.1 \sim 7 \\
n_{\varphi}=0.1 \sim 7
\end{gathered}
$$

phase difference $\alpha^{*}$ is stable under the condition that the value of $\chi$ is negative.

Thus, the residual torque difference can be obtained by

$$
\begin{aligned}
& P_{1}-P_{2}=\left\langle\mu \Phi_{1}\right\rangle=\bar{M}_{e 1}-\bar{R}_{e 1}-\left(\bar{M}_{e 2}-\bar{R}_{e 2}\right) \\
& +\eta_{1} \eta_{2} m r^{2} \omega_{m}^{2} r_{m}\left\{\begin{array}{c}
-\sigma \mu_{11} \sin \alpha+\sigma \mu_{12} \cos (\alpha+\beta) \\
+\mu_{21} \cos (\alpha+\sigma \beta)-\mu_{23} \sin [\alpha+(\sigma+1) \beta]
\end{array}\right\}
\end{aligned}
$$

As $\bar{M}_{e i}, \bar{R}_{e i}(i=1,2)$ are irrelevant to phase difference $\alpha$, in light of (33), the criterion of synchronous stability is expressed

$$
\begin{aligned}
& \chi=\frac{\partial\left(P_{1}-P_{2}\right)}{\partial \alpha} \\
& =\eta_{1} \eta_{2} m r^{2} \omega_{m}^{2} r_{m}\left\{\begin{array}{c}
-\sigma \mu_{11} \cos \alpha-\sigma \mu_{12} \sin (\alpha+\beta) \\
-\mu_{21} \sin (\alpha+\sigma \beta)-\mu_{23} \cos [\alpha+(\sigma+1) \beta]
\end{array}\right\} \\
& <0 .
\end{aligned}
$$

Rearranging the equation above, the criterion of synchronous stability is simplified by

$$
\begin{gathered}
\sigma \mu_{11} \cos \alpha+\sigma \mu_{12} \sin (\alpha+\beta)+\mu_{21} \sin (\alpha+\sigma \beta) \\
+\mu_{23} \cos [\alpha+(\sigma+1) \beta]>0
\end{gathered}
$$

The formula above shows that synchronous stability of the system is also determined by rotation direction of the rotors, coupling coefficients, and installation location of the motors. Only should parameters of the system satisfy the balance equation (30) and the synchronous stability criterion (39), the synchronous operation of the rotors can be implemented. In this case, the phase difference between the rotors is called the stable phase difference.

\section{Numerical Analysis}

The above-mentioned sections have given some theoretical discussions in the simplified form on synchronization problem for the vibration system that the unbalanced rotors are coupled with pendulum. In this section, we will employ some numerical analysis to discuss the stable phase difference, which can be calculated according to (30) and (39). The phase difference is determined by rotation direction of the rotors, coupling coefficients, and installation location of the motors. However, the coupling coefficients are the function of frequency ratios $\left(n_{x} n_{\varphi}\right)$, mass ratios $\left(r_{m}, \eta_{i}\right)$, and motor installation angle $(\beta)$. Therefore, it can be included that the stable phase difference may be influenced by the parameters above. The parameter values corresponding to general engineering application are as given in Table 1 .

6.1. The Rotors Operated in Opposite Direction. The stable phase difference is determined by considering the different values of parameters $\beta, \eta_{i}(i=1,2), n_{x}$, and $n_{\varphi}$. Moreover, in light of (35) and (39), the rotation direction of the two rotors is opposite, and the stable phase difference between the two rotors is shown in Figure 2. According to (20) and (30), we have $\left(-\mu_{11}+\mu_{23}\right) \sin \alpha=0$ when $\sigma=-1$, and the synchronous balance condition as in (17a), (17b), (17c) is simply expressed as $\sin \alpha=0$. Similarly, the synchronous stability criterion can be rewritten by $\left(-\mu_{11}+\mu_{23}\right) \sin \alpha>0$. It then follows that the value of the phase difference is stabilized at 0 [rad] in the blue area, which describes that the synphase motion is stable and the antiphase motion is unstable ([1] describes that the motion, as the existence of $\alpha \in(-\pi / 2, \pi / 2)$, is called synphase synchronization; and the motion, as the existence of $\alpha \in(\pi / 2,3 \pi / 2)$, is called antiphase synchronization). On the contrary, the brown area represents that the synphase synchronization is unstable and the antiphase synchronization is stable. Firstly, installation angle $\beta$ and mass ratio $r_{m}$ are 

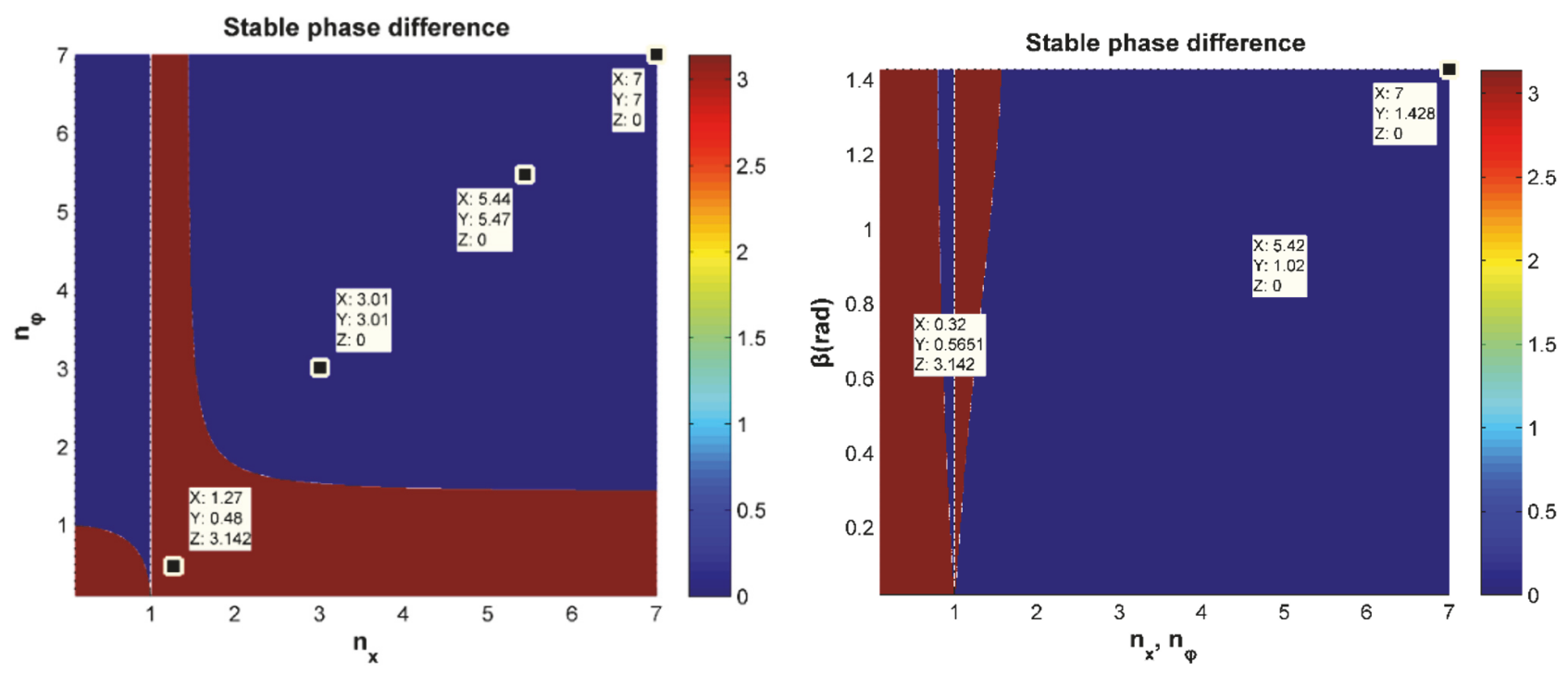

(a) $\eta_{1}=\eta_{2}=1, \eta_{3}=\eta_{4}=12, r_{m}=0.02, \beta=\pi / 3$

(b) $\eta_{1}=\eta_{2}=1, \eta_{3}=\eta_{4}=12, r_{m}=0.02, n_{x} \approx n_{\varphi}$
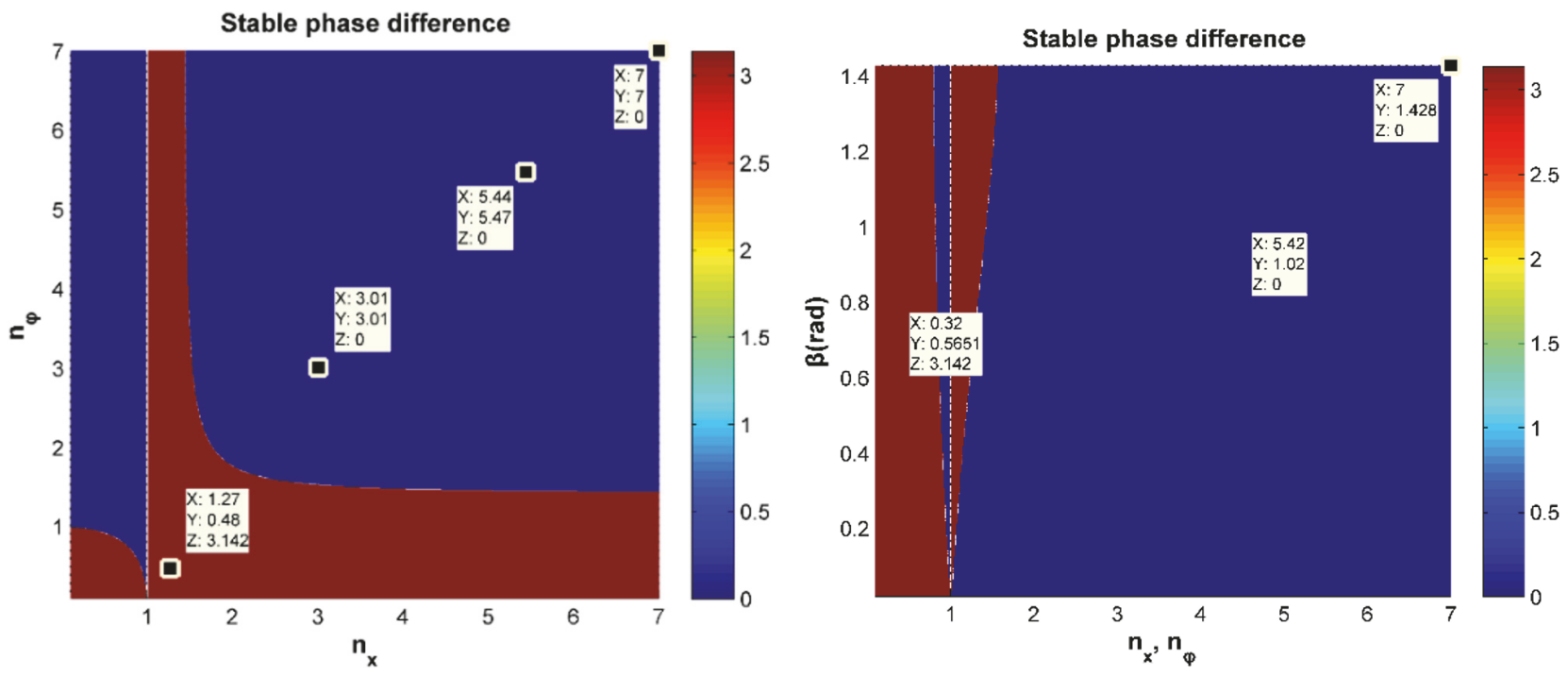

(c) $\eta_{1}=\eta_{2}=1, \eta_{3}=\eta_{4}=12, r_{m}=0.04, \beta=\pi / 3$

(d) $\eta_{1}=0.2, \eta_{2}=1, \eta_{3}=\eta_{4}=12, r_{m}=0.02, n_{x} \approx n_{\varphi}$

FIGURE 2: Stable phase difference when $\sigma=-1$.

fixed at $\pi / 3$ and 0.02 , respectively, and the mass ratios $\left(\eta_{1}, \eta_{2}\right)$ are equal to 1.0. In such values of the parameters, the stable phase difference is shown in Figure 2(a). It is indicated that the stable phase difference is related to frequency ratios $n_{x}$ and $n_{\varphi}$; in other words, the phase difference is dependent on the stiffness of the torque spring in the pendulum and the pressure spring in the vibrating body. Secondly, mass ratio $r_{m}$ is fixed at 0.02 , and the frequency ratios $n_{x}$ and $n_{\varphi}$ are considered identical. Figure 2(b) describes that the stable phase difference is changed with the installation angle $\beta$. It can be seen that the synchronous state of the system is locked in the synphase synchronization when $n_{x}$ and $n_{\varphi}>1.5$, and the variation of installation angle $\beta$ has little influence on the stable phase difference. However, the synchronous state is changed in region of $n_{x}$ and $n_{\varphi}<1.5$. Finally, the effect of mass ratios $r_{m}, \eta_{1}$, and $\eta_{2}$ on the phase difference needs to be discussed. Thus, the parameter values in Figure 2(c) are identical to Figure 2(a) except for $r_{m}$. And the parameter values in Figure 2(d) are identical to Figure 2(b) except for $\eta_{1}$. Comparing Figures 2(c) and 2(d) with Figures 2(a) and 2(b), respectively, it can be found that the values of the stable phase difference are independent of the mass ratios. Therefore, the mass variations of rotors and vibration body would not affect the synchronous motion when the two rotors operate in the opposite direction.

6.2. The Rotors Operated in the Same Direction. In the following calculations, the two rotors rotating in the same direction are considered according to (35) and (39). Firstly, the parameter values in Figure 3(a) are the same as Figure 2(a) except for rotation direction $\sigma$. From Figure 3(a), it follows that two rotors may synchronize either synphase or antiphase, 


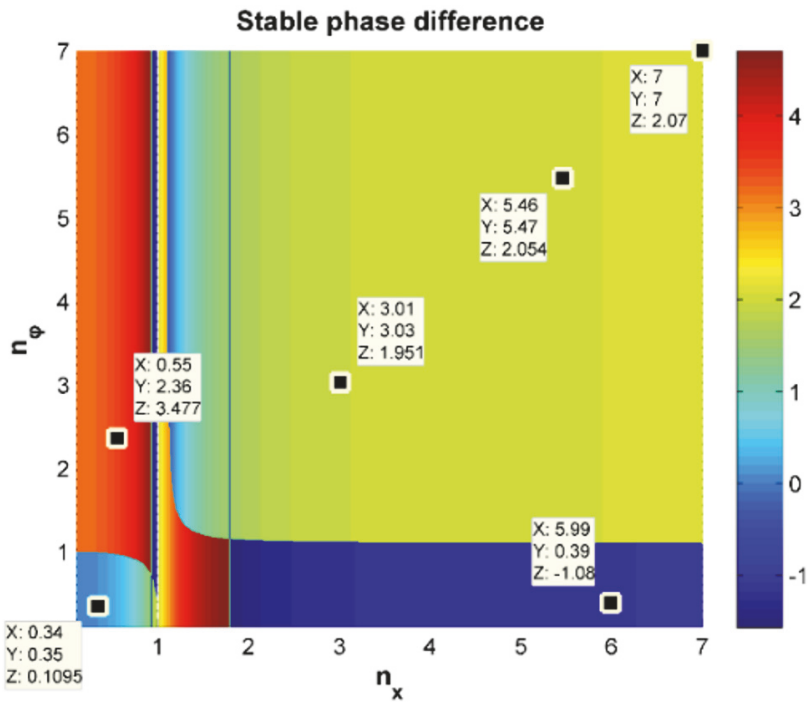

(a) $\eta_{1}=\eta_{2}=1, \eta_{3}=\eta_{4}=12, r_{m}=0.02, \beta=\pi / 3$

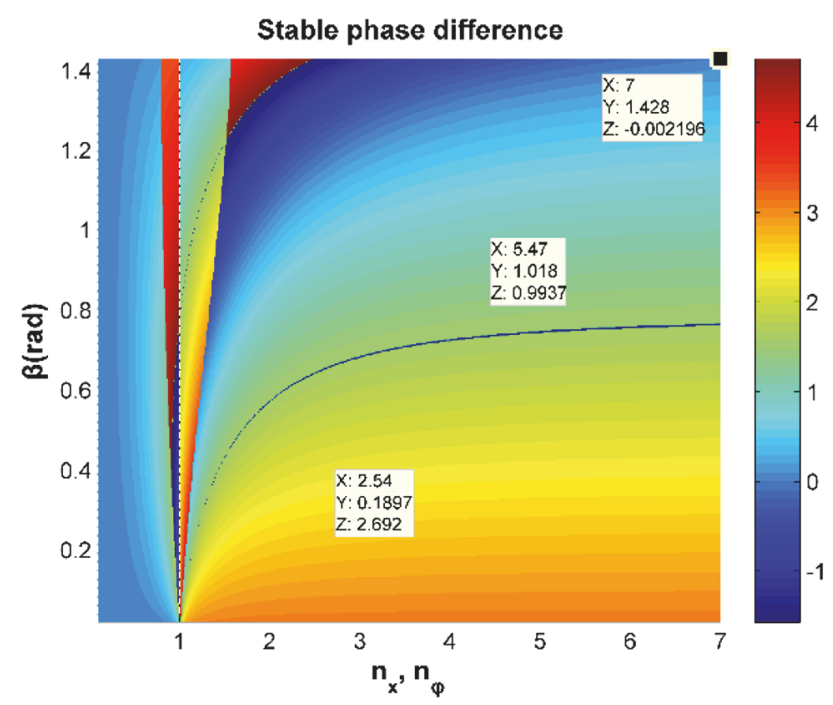

(b) $\eta_{1}=\eta_{2}=1, \eta_{3}=\eta_{4}=12, r_{m}=0.02, n_{x} \approx n_{\varphi}$

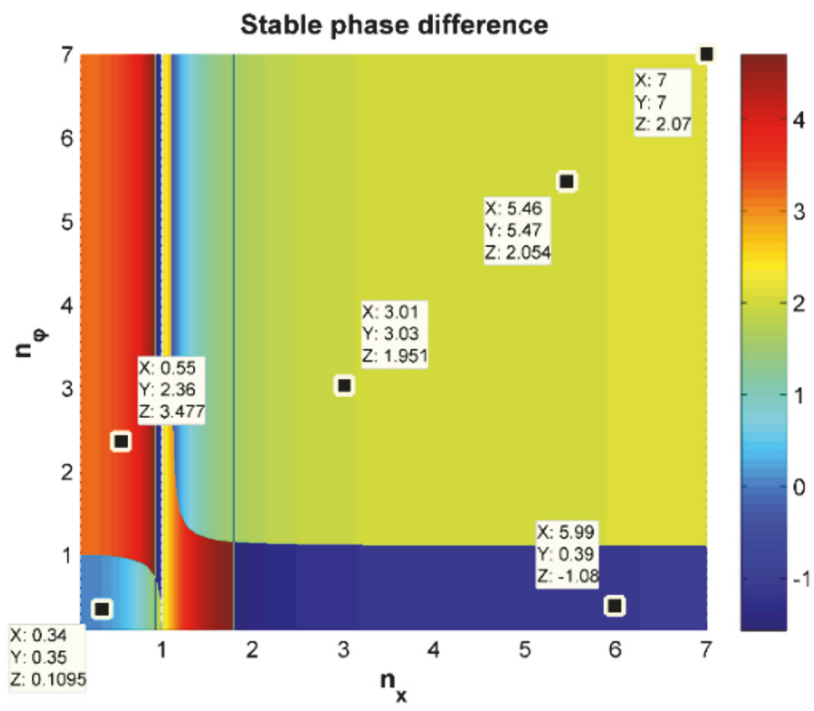

(c) $\eta_{1}=\eta_{2}=1, \eta_{3}=\eta_{4}=12, r_{m}=0.04, \beta=\pi / 3$

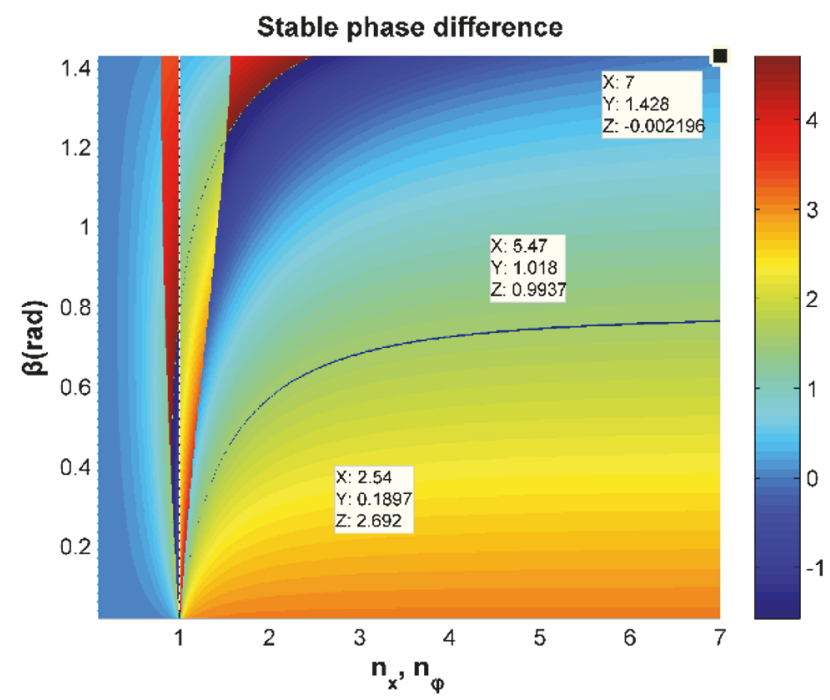

(d) $\eta_{1}=0.2, \eta_{2}=1, \eta_{3}=\eta_{4}=12, r_{m}=0.02, n_{x} \approx n_{\varphi}$

FIGURE 3: Stable phase difference when $\sigma=1$.

also depending on the values of $n_{x}$ and $n_{\varphi}$. The obtained results reveal that, in the interval of $0.1<n_{x}<1$ and $0.1<$ $n_{\varphi}<1$, synphase synchronization is implemented between the rotors; in the interval of $0.1<n_{x}<1$ and $1<n_{\varphi}<$ 7 , antiphase synchronization is implemented between the rotors; in the interval of $1<n_{x}<7,0.1<n_{\varphi}<1$ and $1<n_{x}<$ $7,1<n_{\varphi}<7$, both antiphase and synphase synchronization collectively remained; in the interval of $1<n_{x}<7$ and for $1<n_{\varphi}<7$, the two rotors will be synchronously operated in synphase state. Figure 3(b) shows the variation of the stable phase difference with the installation angle. It is indicated that the synchronous state of the system is obviously influenced by $\beta$. Finally, the effect of mass ratios $r_{m}, \eta_{1}$, and $\eta_{2}$ on the phase difference is discussed. Thus, the parameters in Figure 3(c) are identical with Figure 3(a) except for $r_{m}$, and the parameters in Figure 3(d) are identical with Figure 3(b) except for $\eta_{1}$. Comparing Figures 3(c) and 3(d) with Figures 3(a) and 3(b), respectively, it can be found that the stable phase difference is also independent of the mass ratios. In other words, synchronous state is less influenced by mass of the rotors when the two rotors operate in the same direction.

\section{Sample Verifications}

7.1. For $\sigma=-1, n_{x}=6, n_{\varphi}=6, \eta_{1}=\eta_{2}=1, \eta_{3}=\eta_{4}=12$, $r_{m}=0.02$, and $\beta=\pi / 3$. This subsection refers to the case that the system of after-resonance is coupled with the afterresonance; i.e., $n_{x}=6, n_{\varphi}=6$. The mass of the two rotors operated in opposite direction is identical; i.e., $\eta_{1}=\eta_{2}=1$. In the simulation model, the coefficients of the spring stiffness are $k_{x}=65969[\mathrm{~N} / \mathrm{m}]$ and $k_{\varphi}=1053[\mathrm{~N} \cdot \mathrm{m} / \mathrm{rad}]$, and the other parameter is identical with Table $1(\mathrm{a})$. The numerical 


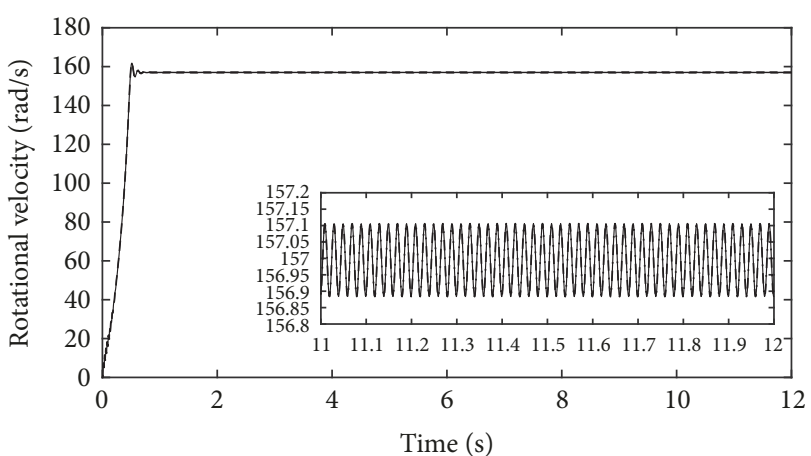

- Motor 1

-- - Motor 2

(a) Velocity

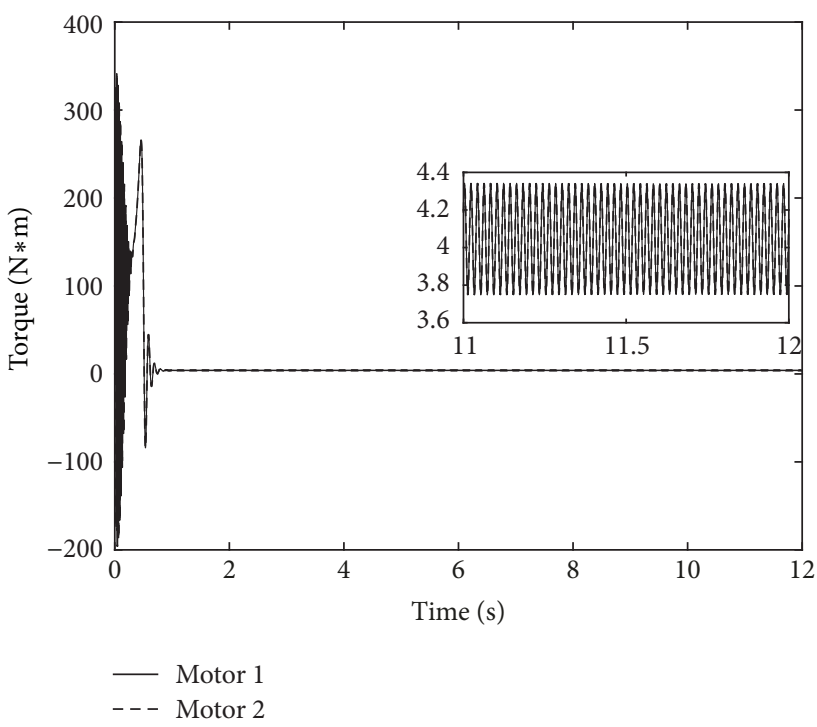

(c) Electromagnetic torque

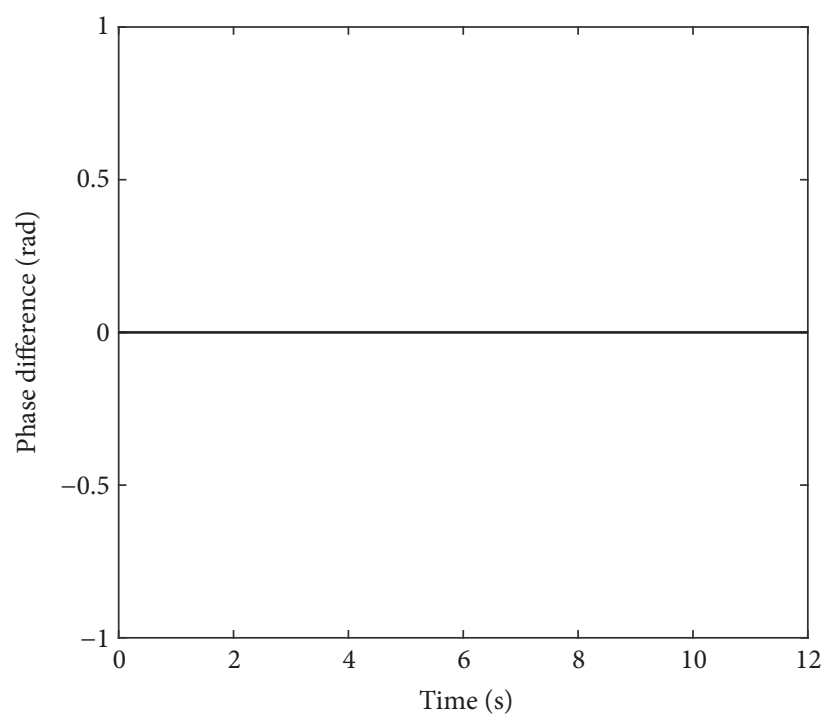

(b) Phase difference
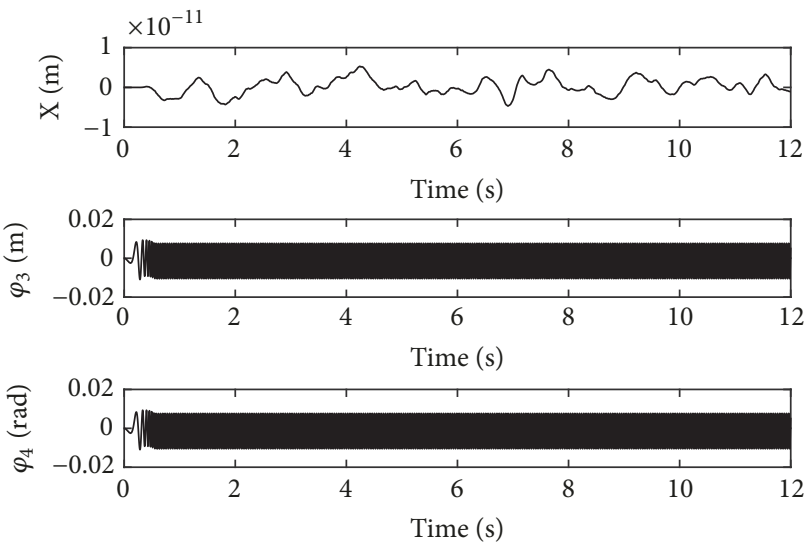

(d) Vibration response

FIgURE 4: The dynamic characteristics when the rotors rotate in the opposite direction.

results of this sample can be calculated through (9a), (9b), $(9 \mathrm{c}),(9 \mathrm{~d}),(9 \mathrm{e})$. When the two motors are supplied by the electric source at the same time, the velocities of the two rotors are compatible (in Figure 4(a)). When the angular velocities of the motors reach the nominal velocity, then the motors oscillate steadily with the identical responses. At this moment, the coupling torques (in Figure 4(c)), making the phase difference $\alpha$ stabilized nearby 0 [rad], are fluctuated. In this case, the two motors rotate stably in synphase synchronization. From Figure 4(c), it follows the displacements of the vibration body and the two motors. It is clear that when the rotation velocity of the two rotors passes through the resonant region of the coupling system, the resonant responses of the system in the $\varphi_{3}$ - and $\varphi_{4}$-directions appear in the starting process. In the synchronous state, the displacements of the vibrating body and the motors are stable, and the amplitudes of them are $0[\mathrm{~m}], 0.01[\mathrm{rad}]$, and $0.01[\mathrm{rad}]$, respectively. Comparing simulation results with Figures 2(a) and 2(b), the stable phase difference obtained by the computer simulation is consistent with the theoretical computation (i.e., the stable phase difference in Figures 3(a) and $3(\mathrm{~b})$ is equal to $0[\mathrm{rad}]$; here, the stable phase difference is fluctuated near $0[\mathrm{rad}])$.

7.2. For $\sigma=1, n_{x}=6, n_{\varphi}=0.3, \eta_{1}=\eta_{2}=1, \eta_{3}=\eta_{4}=12, r_{m}=$ 0.04 , and $\beta=\pi / 3$. Simulation results obtained by (9a), (9b), 

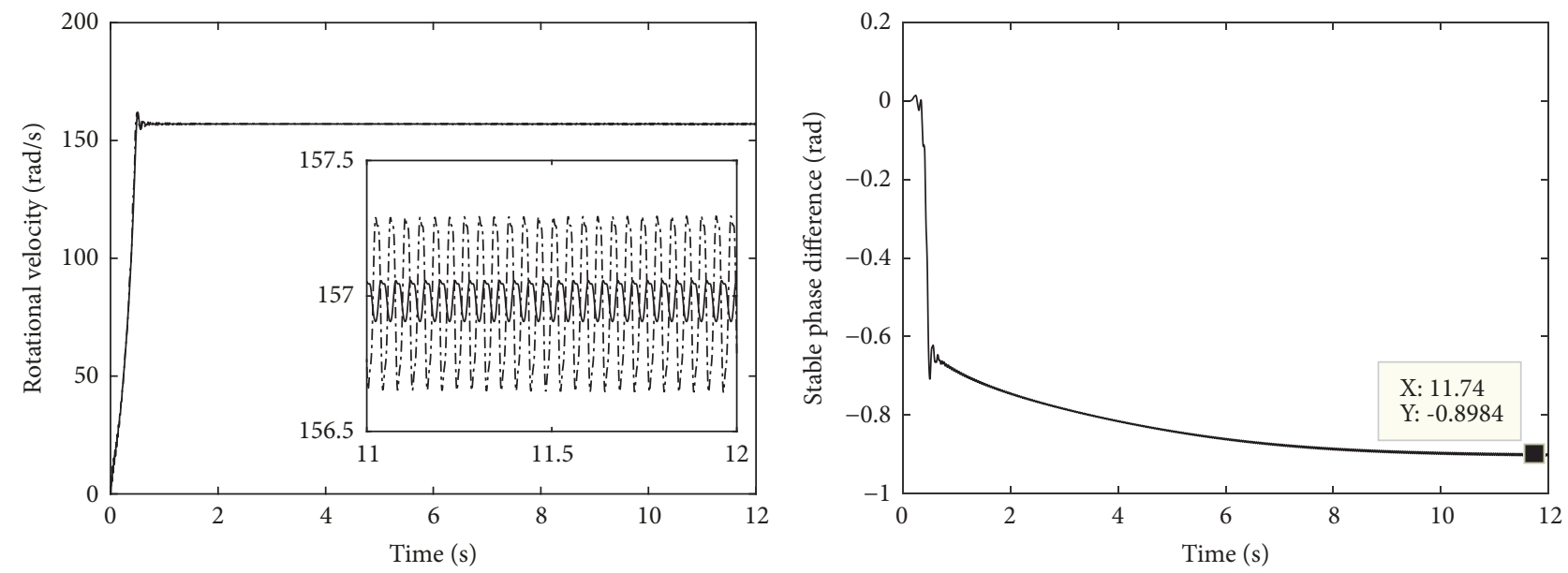

Motor 1

-..- Motor 2

(a) Velocity

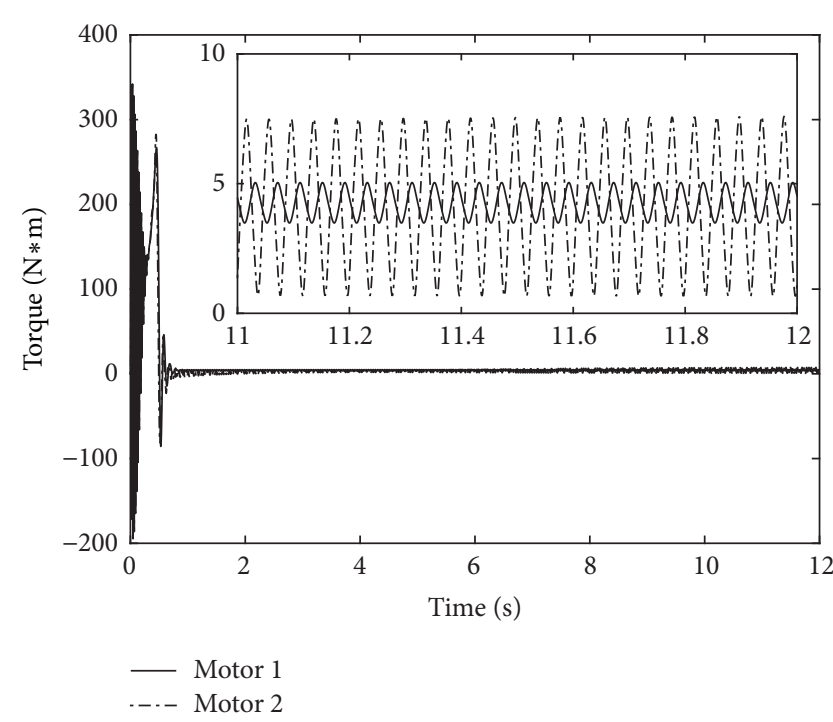

(c) Electromagnetic torque (b) Phase difference
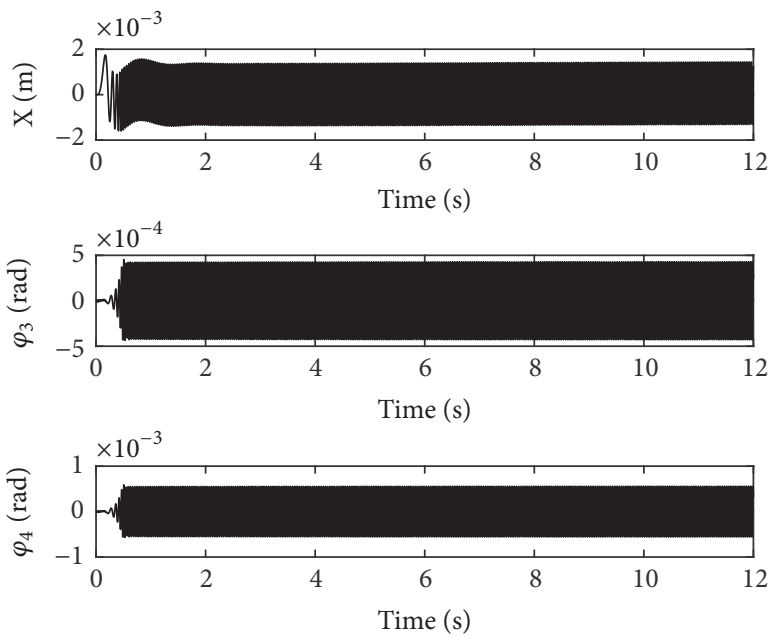

FIGURE 5: The dynamic characteristics when the rotors rotate in the same direction.

(9c), (9d), (9e) are shown in Figure 5 when $\sigma=1, n_{x}=$ $6, n_{\varphi}=0.3, \eta_{1}=\eta_{2}=1, \eta_{3}=\eta_{4}=12, r_{m}=0.04$, and $\beta=\pi / 3$. Here, the coefficients of spring stiffness are $k_{x}=$ $65969[\mathrm{~N} / \mathrm{m}]$ and $k_{\varphi}=976100[\mathrm{~N} \cdot \mathrm{m} / \mathrm{rad}]$, and the rotors are operated in the same direction. The coupling type of the system belongs to type 2 . When the two motors are supplied by the electric source at the same time, the angular accelerations of the two rotors are compatible (in Figure 5(a)). The reason is that the inertia moments of the rotors are identical and the spring stiffness is extremely stronger. During the staring process of the system, the velocity difference exists between the two rotors, which leads to the phase difference instability, shown as in Figure 5(b). However, when the angular velocities of the motors reach the rated speed and the motor oscillate steadily, the synchronization phenomenon occurs. It should be noted that the velocity fluctuation of the motors is smaller than that of Section 7.1, because the vibration of the motors is feebler. At this moment, the average coupling torques (in Figure 5(c)), making the phase difference $\alpha$ stabilized at $0.8984[\mathrm{rad}]$, are approximated to $4.08[\mathrm{~N} \cdot \mathrm{m}]$. In this case, the two motors rotate stably in the synphase synchronization, and the synchronous velocity is $157[\mathrm{rad} / \mathrm{s}]$. From Figure 5(d) it follows the displacements of the vibrating body and the two motors. It can be seen that the displacement responses of the vibrating body and the two motors are stable, and the amplitudes of them are $1.5 \times 10^{-3}[\mathrm{~m}], 4.1 \times 10^{-4}[\mathrm{rad}]$ and $7.3 \times 10^{-4}[\mathrm{rad}]$, respectively. Comparing simulation results with Figure 3(b), it should be noted that the value of the stable phase difference is in agreement with the results obtained for the case of the theoretical solutions (i.e., the stable phase 
difference of such parameters in Figure 3(a) is equal to -1.08 [rad]; here, the stable phase difference is equal to -0.8984 $[\mathrm{rad}])$.

\section{Conclusions}

In this paper, the dual-pendulum-rotor system is concerned. The research results show that the spring stiffness, the installation angular of the motor, and rotation direction of the rotors have a large influence on the existence and stability of the synchronization state in the coupling system, and the mass ratios of the system are irrelevant to the synchronous state of the system. It should be noted that to ensure the implementation of the synchronization of the system, the values of the parameters of the system should be far away to the two "critical points". To verify the correctness of the theoretical computations, some example simulations are preformed, and the results of theoretical computation are in accordance with that of example simulations.

The dual-pendulum-rotor system widely appears in aeropower plant, mining screening machines, parallel robots, and the like of the other rotation equipment. However, the dynamic characteristics and performance accuracy of the dual-pendulum-rotor system are mainly influenced by synchronous behavior between the rotors. In the early stage, for the developing and understanding the internal characteristics of the system, we only consider the vibrating body under the assumption of horizontal displacement. What is synchronization state of the system that the vibrating body simultaneously vibrates in the pitch and vertical directions? We believe that finding the answer to this question is the next step in challenging task of getting a complete understanding of synchronization in such system.

\section{Data Availability}

The data used to support the findings of this study are available from the corresponding author upon request.

\section{Conflicts of Interest}

The authors declare that there are no conflicts of interest regarding the publication of this paper.

\section{Acknowledgments}

This study is supported by National Natural Science Foundation of China (Grant no. 51705437), scientific research starting project of SWPU (no. 2017QHZ009), and the plan of science and technology of Sichuan province (no. 2016RZ0059).

\section{References}

[1] I. I. Blekhman, Synchronization in science and technology, ASME Press, New York, USA, 1988.

[2] I. I. Blekhman, Selected Topics in Vibrational Mechanics World scientific, Singapore, 2002.
[3] C. Murguia, R. H. B. Fey, and H. Nijmeijer, "Synchronization of identical linear systems and diffusive time-delayed couplings," IEEE Transactions on Circuits and Systems I: Regular Papers, vol. 61, no. 6, pp. 1801-1814, 2014.

[4] A. Arenas, A. Dì az-Guilera, J. Kurths, Y. Moreno, and C. Zhou, "Synchronization in complex networks," Physics Reports, vol. 469, no. 3, pp. 93-153, 2008.

[5] M. A. Barrón and M. Sen, "Synchronization of four coupled van der Pol oscillators," Nonlinear Dynamics, vol. 56, no. 4, pp. 357$367,2009$.

[6] R. Yamapi and P. Woafo, "Dynamics and synchronization of coupled self-sustained electromechanical devices," Journal of Sound and Vibration, vol. 285, no. 4-5, pp. 1151-1170, 2005.

[7] J. F. Totz, R. Snari, D. Yengi, M. R. Tinsley, H. Engel, and K. Showalter, "Phase-lag synchronization in networks of coupled chemical oscillators," Physical Review E: Statistical, Nonlinear, and Soft Matter Physics, vol. 92, no. 2, Article ID 022819, 2015.

[8] A. Pikovsky, M. Rosenblum, and J. Kurths, Synchronization: A Universal Concept in Nonlinear Sciences, Cambridge unverisity press, Cambridge, UK, 2001.

[9] P. Koluda, P. Perlikowski, K. Czolczynski, and T. Kapitaniak, "Synchronization configurations of two coupled double pendula," Communications in Nonlinear Science and Numerical Simulation, vol. 19, no. 4, pp. 977-990, 2014.

[10] R. Dilão, "Anti-phase synchronization and ergodicity in arrays of oscillators coupled by an elastic force," The European Physical Journal Special Topics, vol. 223, no. 4, pp. 665-676, 2014.

[11] L. Marcheggiani, R. Chacón, and S. Lenci, "On the synchronization of chains of nonlinear pendula connected by linear springs," The European Physical Journal Special Topics, vol. 223, no. 4, pp. 729-756, 2014.

[12] R. A. O. Signeul, "Vibrating device for a directed vibratory effected by means of rotatable vibratory members," United states patent, No.2,531,706, 1950.

[13] B. C. Wen, J. Fan, and C. Y. Zhao, Synchronization and controled sychronization in engineering, Science press, Beijing, China, 2009.

[14] C. Zhao, B. Wen, and X. Zhang, "Synchronization of the four identical unbalanced rotors in a vibrating system of plane motion," Science China Technological Sciences, vol. 53, no. 2, pp. 405-422, 2010.

[15] C. Y. Zhao, H. T. Zhu, R. Z. Wang, and B. C. Wen, "Synchronization of two non-identical coupled exciters in a non-resonant vibrating system of linear motion. Part I: theoretical analysis," Shock and Vibration, vol. 16, no. 5, pp. 505-515, 2009.

[16] J. M. Balthazar, J. L. Felix, and R. M. Brasil, "Some comments on the numerical simulation of self-synchronization of four nonideal exciters," Applied Mathematics and Computation, vol. 164, no. 2, pp. 615-625, 2005.

[17] A. A. N. Djanan, B. R. N. Nbendjo, and P. Woafo, "Effect of selfsynchronization of DC motors on the amplitude of vibration of a rectangular plate," The European Physical Journal Special Topics, vol. 223, no. 4, pp. 813-825, 2014.

[18] X. L. Zhang, B. C. Wen, and C. Y. Zhao, "Synchronization of three non-identical coupled exciters with the same rotating directions in a far-resonant vibrating system," Journal of Sound and Vibration, vol. 332, no. 9, pp. 2300-2317, 2013.

[19] X. Zhang, B. Wen, and C. Zhao, "Theoretical study on synchronization of two exciters in a nonlinear vibrating system with multiple resonant types," Nonlinear Dynamics, vol. 85, no. 1, pp. 141-154, 2016. 
[20] P. Fang and Y. Hou, "Synchronization characteristics of a rotor-pendula system in multiple coupling resonant systems," Proceedings of the Institution of Mechanical Engineers, Part C: Journal of Mechanical Engineering Science, vol. 232, no. 10, pp. 1802-1822, 2017.

[21] L. Tang, X. Zhu, and J. Li, "Effects of the Synchronous Variation of the Static and the Kinetic Friction Coefficients on Stick-Slip Vibration of Drillstring," Iranian Journal of Science and Technology, Transactions of Mechanical Engineering.

[22] W. Craig, D. Yeo, and D. A. Paley, "Dynamics of a rotorpendulum with a small, stiff propeller in wind," in Proceedings of the ASME 2016 Dynamic Systems and Control Conference, DSCC 2016, USA, October 2016.

[23] X. Zhang, B. Wen, and C. Zhao, "Vibratory synchronization transmission of a cylindrical roller in a vibrating mechanical system excited by two exciters," Mechanical Systems and Signal Processing, vol. 96, pp. 88-103, 2017.

[24] P. Fang, Y. Hou, Y. Nan, and L. Yu, "Study of synchronization for a rotor-pendulum system with Poincare method," Journal of Vibroengineering, vol. 17, no. 5, pp. 2681-2695, 2015.

[25] P. Fang, Y. Hou, L. Zhang, M. Du, and M. Zhang, "Synchronous behavior of a rotor-pendulum system," Acta Physica Sinica, pp. 254-265, 2016.

[26] Y. Hou and P. Fang, "Investigation for Synchronization of a Rotor-Pendulum System considering the Multi-DOF Vibration," Shock and Vibration, vol. 2016, Article ID 8641754, 22 pages, 2016.

[27] Y. Hou, M. Du, P. Fang, and L. Zhang, "Synchronization and stability of an elastically coupled tri-rotor vibration system," Journal of Theoretical and Applied Mechanics (Poland), vol. 55, no. 1, pp. 227-240, 2017. 


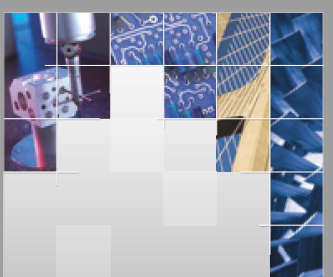

\section{Enfincering}
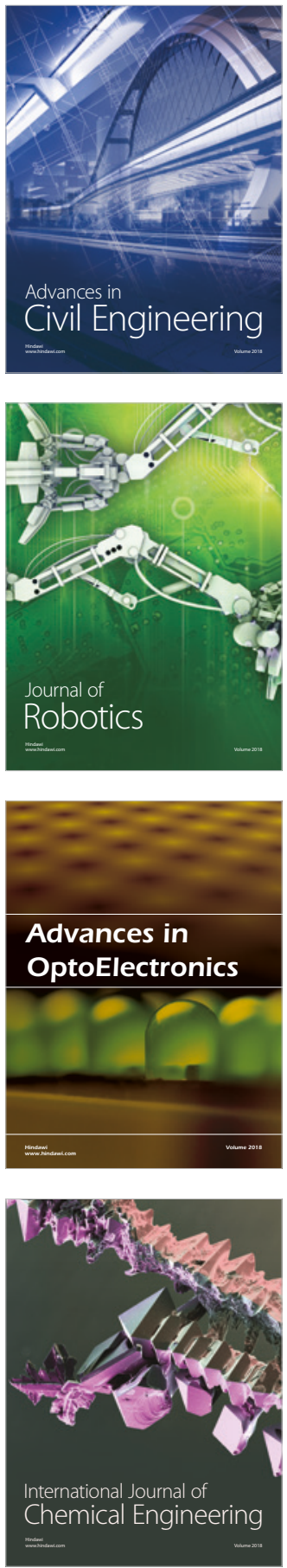

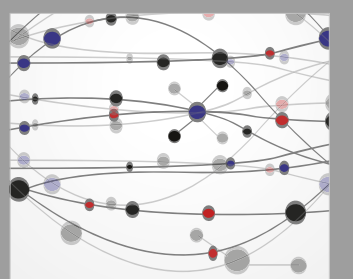

\section{Rotating \\ Machinery}

The Scientific World Journal

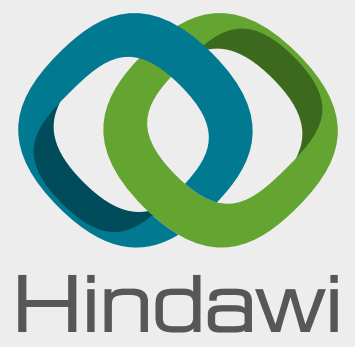

Submit your manuscripts at

www.hindawi.com
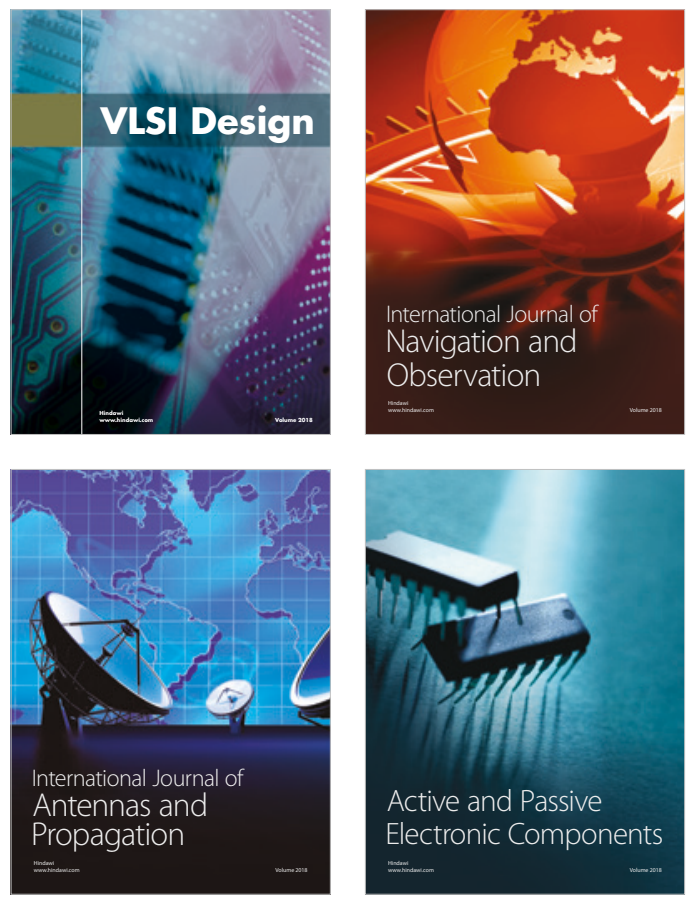
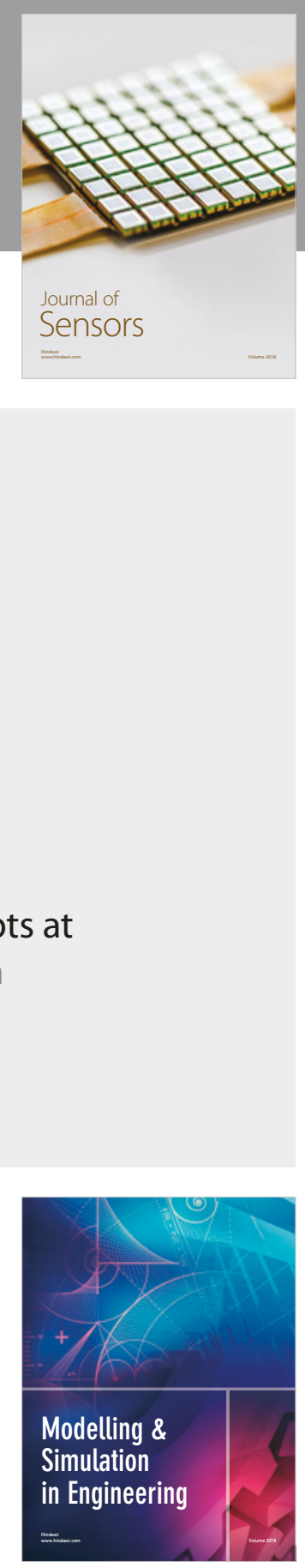

\section{Advances \\ Multimedia}
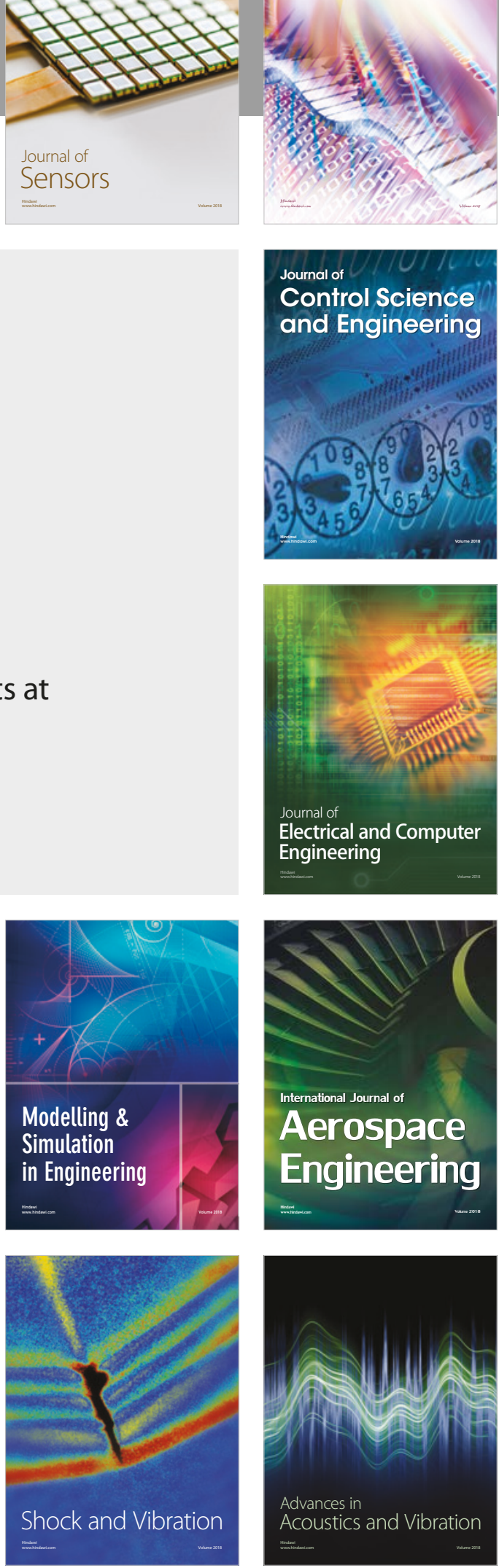